\title{
FINITE GENERATION OF EQUIVARIANT COHOMOLOGY FOR A p-COMPACT GROUP $G$
}

\author{
HYANG - SOOK LEE
}

For a $p$-compact group $G$ and $G$-space $X$, we prove the finite generation of the equivariant cohomology $H_{G}^{*}(X)$ and give the form of the Poincaré series of $H_{G}^{*}(X)$.

\section{0 . INTRODUCTION}

For a compact Lie group $G$, the algebra $H_{G}^{*}=H^{*}\left(B G, \mathbb{F}_{p}\right)$ is finitely generated. This result is extended to finite loop spaces and other loop spaces called $p$-compact groups [2]. Dwyer and Wilkerson introduced p-compact groups and proved lots of their properties in detail [2]. Their work shows that a $p$-compact group has much of the rich internal structure of a compact Lie group. In [3], Quillen proved finite generation of $H_{G}^{*}(X)=H^{*}\left(E G \times_{G} X\right)$ for the general equivariant cohomology ring of a $G$-space $X$, where $G$ is a compact Lie group. In this paper, we generalise theorems on finite generation of $H_{G}^{*}(X)$ for a $p$-compact group $G$ and $G$-space $X$. We also give the form of the Poincaré series of $H_{G}^{*}(X)$. In the first section, we give brief definitions and properties as background. In Section 2, we give the main results.

\section{Preliminaries}

A graded vector space $H^{*}$ over a field $F$ is finite dimensional if each $H^{i}$ is finite dimensional over $F$ and $H^{i}=0$ for all but finite number of $i$. A space $X$ is $\mathbb{F}_{p}$-finite if $H^{*} X$ is finte dimensional over the finite field $\mathbb{F}_{p}$. Let $\varepsilon_{X}: X \rightarrow X_{p}^{-}$be a natural map for any space $X$ where $(-)_{p}^{-}$is the $\mathbb{F}_{p}$-completion functor constructed by Bousfield and Kan [1]. If $\varepsilon_{X}$ is a homotopy equivalence, we say $X$ is $\mathbb{F}_{p}$-complete.

DEFinition 1.1: A p-compact group is a loop space $G$ satisfying the following equivalent conditions.

(1) $G$ is $\mathbb{F}_{p}$-finite, $\mathbb{F}_{p}$-complete and $\pi_{0} G$ is a finite $p$-group.

(2) $G$ is $\mathbb{F}_{p}$-finite and $B G$ is $\mathbb{F}_{p}$-complete.

Received 19th February, 1998

The author was supported by Ministry of Education, BSRI - 96 - 1424, partially supported by KOSEF 97-07-01-02-01-5.

Copyright Clearance Centre, Inc. Serial-fee code: 0004-9729/98 $\$ A 2.00+0.00$. 
TheOREM 1.2. [2] If $G$ is a p-compact group, then $H^{*}\left(B G, \mathbb{F}_{p}\right)$ is finitely generated as an algebra.

From classical algebra, if $X$ is connected then $H^{*} X$ is finitely generated as an algebra if and only if $H^{*} X$ is Noetherian as a graded ring if and only if every graded ideal in $H^{*} X$ has a finite number of homogeneous generators if and only if every graded submodule of a graded finitely generated $H^{*} X$-module is itself finitely generated. Also a $H^{*} X$-module satisfies the ascending chain condition on submodules if and only if every submodule of $H^{*} X$-module is finitely generated.

A homomorphism $f: G \rightarrow K$ of $p$-compact groups is a pointed map $B f: B G \rightarrow B K$. The homogeneous space $K / G$ is defined to be the homotopy fibre of $B f$ over the basepoint of $B K$. The homomorphism $f$ is said to be a monomorphism if $K / G$ is $\mathbb{F}_{p}$-finite and an epimorphism if $\Omega(K / G)$ is a p-compact group. A short exact sequence $G \stackrel{f}{\longrightarrow} H \stackrel{g}{\longrightarrow} K$ of $p$-compact groups is a sequence such that $B G \rightarrow B H \rightarrow B K$ is a fibration sequence where $f$ is a monomorphism and $g$ is an epimorphism.

Let $G$ be a $p$-compact group and $X$ be a $G$-space defined to be a fibration $E G{ }{ }_{G} X \rightarrow$ $B G$ with $X$ as the fibre. Here $E G$ is the universal bundle over $B G$. The equivariant cohomology of the $G$-space $X$ is defined by the formula

$$
H_{G}^{*}(X)=H^{*}\left(E G \times_{G} X\right)
$$

where $H^{*}(-)$ means ordinary cohomology.

A morphism $(f, u):(G, X) \rightarrow\left(G^{\prime}, X^{\prime}\right)$ is a homomorphism of $p$-compact groups $f: G \rightarrow G^{\prime}$ and for a $G$-space $X$ and $G^{\prime}$-space $X^{\prime}$, a map $u: X \rightarrow X^{\prime}$ which is $f$ equivariant, that is, $u(g x)=f(g) u(x)$. Let $E G \rightarrow B G$ and $E G^{\prime} \rightarrow B G^{\prime}$ be the principal $G$ and $G^{\prime}$-bundle respectively, and consider the following diagram

where $p_{1}$ and $p_{2}$ are induced by the projections of $E G \times E G^{\prime}$ onto its factors, $\bar{u}$ is induced by $u$ and $v: E G \rightarrow E G^{\prime}$ is an $f$-equivariant map, $\overline{v \times u}$ is the map on orbit spaces induced by $v \times u$. This diagram yields a canonical homomorphism

$$
\left(p_{1}^{*}\right)^{-1} p_{2}^{*} \bar{u}^{*}: H^{*}\left(E G^{\prime} \times_{G^{\prime}} X^{\prime}\right) \rightarrow H^{*}\left(E G \times_{G} X\right)
$$

This homomorphism will be denoted by

$$
(f, u)^{*}: H_{G^{\prime}}^{*}\left(X^{\prime}\right) \rightarrow H_{G}^{*}(X)
$$

If $X$ is a point, we write $H_{G}^{*}(p t)=H^{*}(B G)=H_{G}^{*}$. 


\section{THE FINITENESS THEOREM}

Let $G$ be a $p$-compact group and $X$ be a $G$-space. In this section we study the finite generation of $H_{G}^{*}(X)$. The coefficient ring is assumed to be a finite field $\mathbb{F}_{p}$.

LEMMA 2.1. [2] If $G$ is a p-compact group and $M$ is a $\mathbb{F}_{p}$-vector space which is a module over $\pi_{1} B G$, then $H^{*}(B G, M)$ is finitely generated as a module over $H^{*} B G$.

THEOREM 2.2. [2] A homomorphism $f: G \rightarrow K$ of $p$-compact groups is a monomorphism if and only if the ring $H_{G}^{*}$ is a finitely generated module over $H_{K}^{*}$.

Let $f: G \rightarrow K$ be a monomorphism of $p$-compact groups. We consider $H_{G}^{*}(X)$ as an algebra over $H_{K}^{*}$ by means of the homomorphism $(f, \rho)^{*}$ where $\rho$ is the map from $X$ to a point. Then we have the following theorem.

THEOREM 2.3. If $X$ is $\mathbb{F}_{p}$-finite, then $H_{G}^{*}(X)$ is a finitely generated $H_{K}^{*}$-module.

Proof: We consider the Serre spectral sequence for the $E_{2}$-term of the fibre space $E G \times{ }_{G} X \rightarrow B G$

$$
E_{2}^{s, t}=H^{s}\left(B G, H^{t}(X)\right) \Longrightarrow H_{G}^{s+t}(X)
$$

Now $X$ is $\mathbb{F}_{p}$-finite, hence the $E_{2}$-term is finitely generated as a module over $H_{G}^{*}$ by Lemma 2.1. Since $H_{G}^{*}$ is a Noetherian ring, $E_{\tau}$ is also a finitely generated $H_{G}^{*}$-module by induction on $r . E_{r}=E_{\infty}$ for sufficiently large $r$, and hence $H_{G}^{*}(X)$ is a finitely generated module over $H_{G}^{*}$. But $H_{G}^{*}$ is a finitely generated $H_{K}^{*}$-module (Theorem 2.2). Therefore $H_{G}^{*}(X)$ is a finitely generated module over $H_{K}^{*}$.

Corollary 2.4. $H_{G}^{*}(X)$ is a finitely generated algebra over $\mathbb{F}_{p}$.

A homomorphism $\zeta: R \rightarrow S$ of graded commutative rings is finite if $S$ is a finitely generated module over $R$ via $\zeta$.

Corollary 2.5. If $(f, u):(G, X) \rightarrow\left(G^{\prime}, X^{\prime}\right)$ is a morphism such that $f$ is a monomorphism and $X$ is $\mathbb{F}_{p}$-finite, then $(f, u)^{*}: H_{G^{\prime}}^{*}\left(X^{\prime}\right) \rightarrow H_{G}^{*}(X)$ is finite.

Proof: If we choose a monomorphism $G^{\prime} \rightarrow K$, then $H_{G}^{*}(X)$ is a finitely generated module over $H_{K}^{*}$, hence also a finitely generated module over $H_{G^{\prime}}^{*}(X)$.

Now we recall the Euler-Poincaré function $\varphi$ which maps certain modules to elements of an Abelian group and satisfies the following condition;

If $0 \rightarrow M^{\prime} \rightarrow M \rightarrow M^{\prime \prime} \rightarrow 0$ is exact, then $\varphi(M)$ is defined if and only if $\varphi\left(M^{\prime}\right)$ and $\varphi\left(M^{\prime \prime}\right)$ are defined, and $\varphi(M)=\varphi\left(M^{\prime}\right)+\varphi\left(M^{\prime \prime}\right)$.

Remark. Assume $\varphi$ is defined on finite dimensional vector spaces over $\mathbb{F}_{p}$, and is equal to the dimension. Then the values of $\varphi$ are in the additive group of integers.

If $X$ is $\mathbb{F}_{p}$-finite, the Poincare series of $H_{G}^{*}(X)$ is defined by

$$
P_{t}\left(H_{G}^{*}(X)\right)=\sum_{i=0}^{\infty}\left(\operatorname{dim}_{F_{p}} H_{G}^{i}(X)\right) t^{i}
$$

If $H_{G}^{*}(X)$ is finite dimensional over $\mathbb{F}_{p}$, then $P_{t}\left(H_{G}^{*}(X)\right)$ is a polynomial. 
If $X$ is $\mathbb{F}_{p}$-finite, we showed $H_{G}^{*}(X)$ is a finitely generated $H_{K}^{*}$-module in Theorem 2.3. Let $m$ be the number of generators of $H_{K}^{*}$ as an algebra over $\mathbb{F}_{p}$. Then we give the following type of Poincaré series of $H_{G}^{*}(X)$.

Proposition 2.6. The Poincaré series of $H_{G}^{*}(X)$ is a rational function of the form

$$
P_{t}\left(H_{G}^{*}(X)\right)=\frac{f(t)}{\prod_{i=0}^{m}\left(1-t^{d_{i}}\right)}
$$

where $d_{i}$ 's are the corresponding degrees of generators of $H_{K}^{*}$ and $f(t)$ is a polynomial with integer coefficients.

Proof: We use induction on $m$. For $m=0, H_{G}^{*}(X)$ is a finitely generated $\mathbb{F}_{p^{-}}$ module, and hence $P_{t}\left(H_{G}^{*}(X)\right)=f(t)$ is a polynomial in $\mathbb{Z}[t]$. Assume $m \geqslant 1$. Since $H_{K}^{*}$ is a finitely generated algebra over $\mathbb{F}_{p}$, we set $H_{K}^{*}=\mathbb{F}_{p}\left\langle x_{1}, x_{2}, \cdots, x_{m}\right\rangle$ where $\operatorname{deg} x_{i}=$ $d_{i} \geqslant 1$. We consider the following exact sequence by multiplying $x_{m}$ on $H_{G}^{*}(X)$

$$
0 \rightarrow C_{n} \rightarrow H_{G}^{n}(X) \stackrel{x_{m}}{\longrightarrow} H_{G}^{n+d_{m}}(X) \rightarrow L_{n+d_{m}} \rightarrow 0 .
$$

Let $C=\oplus_{n} C_{n}$ and $L=\oplus_{n} L_{n}$. Then $C$ and $L$ are finitely generated $H_{K}^{*}$-modules (as a submodule and factor module respectively) and annihilated by $x_{m}$, hence are graded $\mathbb{F}_{p}\left\langle x_{1}, x_{2}, \cdots, x_{m-1}\right\rangle$-modules. By the Remark,

$$
\operatorname{dim} C_{n}-\operatorname{dim} H_{G}^{n}(X)+\operatorname{dim} H_{G}^{n+d_{m}}(X)-\operatorname{dim} L_{n+d_{m}}=0 .
$$

Multiplying by $t^{n+d_{m}}$ and summing over $n$,

$$
\begin{aligned}
\sum_{n=0} t^{n+d_{m}} \operatorname{dim} C_{n} & -\sum_{n=0} t^{n+d_{m}} \operatorname{dim} H_{G}^{n}(X) \\
& +\sum_{n=0} t^{n+d_{m}} \operatorname{dim} H_{G}^{n+d_{m}}(X)-\sum_{n=0} t^{n+d_{m}} \operatorname{dim} L_{n+d_{m}} \\
=0 . &
\end{aligned}
$$

Hence

$$
\begin{aligned}
t^{d_{m}} \cdot\left(\sum_{n=0} \operatorname{dim} C_{n} \cdot t^{n}\right)-t^{d_{m}} \cdot\left(\sum_{n=0} \operatorname{dim} H_{G}^{n}(X) \cdot t^{n}\right) \\
\quad+\sum_{n=0} \operatorname{dim} H_{G}^{n+d_{m}}(X) \cdot t^{n+d_{m}}-\sum_{n=0} \operatorname{dim} L_{n+d_{m}} \cdot t^{n+d_{m}} \\
=t^{d_{m}} \cdot P_{t}(C)-t^{d_{m}} \cdot P_{t}\left(H_{G}^{*}(X)\right)+P_{t}\left(H_{G}^{*}(X)\right)-P_{t}(L)-g(t) \\
=0
\end{aligned}
$$

where

$$
g(t)=\sum_{i=0}^{d_{m}-1} \operatorname{dim} H_{G}^{i}(X) \cdot t^{i}-\sum_{i=0}^{d_{m}-1} \operatorname{dim} L_{i} \cdot t^{i} \in \mathbb{Z}[t]
$$


Then

$$
\begin{aligned}
\left(1-t^{d_{m}}\right) P_{t}\left(H_{G}^{*}(X)\right) & =P_{t}(L)-t^{d_{m}} P_{t}(C)+g(t) \\
& =\frac{f_{1}(t)}{\prod_{i=1}^{m-1}\left(1-t^{d_{i}}\right)}-\frac{t^{d_{m}} \cdot f_{2}(t)}{\prod_{i=1}^{m-1}\left(1-t^{d_{i}}\right)}+g(t)
\end{aligned}
$$

by induction. Therefore

$$
\begin{aligned}
P_{t}\left(H_{G}^{*}(X)\right) & =\frac{f_{1}(t)-t^{d_{m}} \cdot f_{2}(t)+g(t) \prod_{i=1}^{m-1}\left(1-t^{d_{i}}\right)}{\prod_{i=1}^{m}\left(1-t^{d_{i}}\right)} \\
& =\frac{f(t)}{\prod_{i=1}^{m}\left(1-t^{d_{i}}\right)}
\end{aligned}
$$

where $f(t) \in \mathbb{Z}[t]$.

\section{REFERENCES}

[1] A.K. Bousfield and D.K. Kan, Homotopy limits, completions and localizations, Lecture Notes in Math. 304 (Springer-Verlag, Berlin, 1972).

[2] W.G. Dwyer and C.W. Wilkerson, 'Homotopy fixed point methods for Lie groups and finite loop spaces', Ann. of Math. 139 (1994), 395-442.

[3] D. Quillen, 'The spectrum of an equivariant cohomology ring I', Ann. of Math. 94 (1991), 549-572.

Department of Mathematics

Ewha Women's University

Seoul $120-750$

Korea

e-mail: hsl@mm.ewha.ac.kr 